\title{
EDITORIAL
}

\section{ARTICLES PUBLISHED IN THE SEPTEMBER/OCTOBER 2010 ISSUE OF THE "REVISTA DO INSTITUTO DE MEDICINA TROPICAL DE SÃO PAULO"}

In the September/October 2010 issue of "Revista do Instituto de Medicina Tropical de S. Paulo" (Journal of the São Paulo Institute of Tropical Medicine) CABRERA et al. reported an epidemiological investigation of acute Chagas disease in a non-endemic area of the Peruvian Amazon Region. A seroepidemiological survey was carried out in relatives of the patient and triatomine bugs were searched for inside houses, peridomicilliary and wild environments. Panstrongylus geniculatus and Rhodnius pictipes were found but the source of transmission remained unknown.

WOODS et al. reported a ten years experience with Jorge Lobo's disease in the state of Acre, Amazon Region, Brazil. A retrospective study was carried out based on the analysis of clinical records, and 249 cases of the disease were reported; 30 were females and 219 males. Of these patients 153 had localized lesions, 94 of them on one ear, 55 multifocal lesions and 41 disseminated lesions. The average time between the onset of symptoms and diagnosis was 19 years.

CHIEFFI et al. studied on behavioral changes in Rattus norvegicus experimentally infected by Toxocara canis larvae. Fifty rats were used divided into three groups: G1, 20 rats infected with $300 \mathrm{~T}$. canis eggs; G2, 20 rats infected with 2000 eggs; G3 non infected rats. Thirty and 60 days post-infection, rats from all groups were submitted to an open-field and subsequently to an elevated plus-maze apparatus, for five minutes each. Data indicated improvement in mobility and exploratory behavior in infected rats, principally in G2, which provides support for the hypothesis that behavioral alterations in infected rodents enhances the transmission rate of the ascarid to dogs.

LIMA VERDE et al. reported hyponatremia and serum hypoosmolality in 55 kala-azar patients (20 normal individuals as a control group). High first morning osmolality and high 24 hour-urine osmolality demonstrated persistent antidiuretic hormone secretion. Urinary sodium was high. Low seric uric acid with increased fractional uric acid excretion occurred roughly in $2 / 3$ of patients. Normal ADH levels were observed in kala-azar patients and no endocrine or renal dysfunction was noted. It is possible that most hyponatremic kala-azar patients present the syndrome of inappropriate antidiuretic hormone secretion.

CORTI et al. reported for the first time a rupioid eruption in an AIDS patient. Disseminated histoplasmosis is a relatively common AIDSdefining illness and a broad spectrum of clinical skin lesions associated to this mycosis have been described but so far rupioid histoplasmosis had never been seen before.

CUNHA et al. evaluated and compared the pathogenicity of rabies virus isolates obtained from bats and dogs. For evaluation of pathogenicity mice were inoculated by subcutaneous route and challenged through intracerebral (IC) route, when isolates proved to be fully pathogenic. When inoculated only by intramuscular route the pathogenicity observed showed different death rates: 60.05 for the Desmodus rotundus and 5.2\% for the Eptesicus furinalis isolates. Mice receiving one dose of vaccine were partially protected against the dog isolate. The isolates from bats were pathogenic by the IC route in mice but showed different degrees of pathogenicity when inoculated through the intramuscular route. Results suggest that the commercial vaccine protects mice from infection with bat rabies virus isolates in addition to a canine rabies virus isolate.
SILVA \& EVANGELISTA conducted a study of syndrome surveillance in patients with acute febrile illness suspicious of dengue but with negative serology. Serum samples were collected from 144 people during convalescence. All the exams were re-tested for dengue which was confirmed in $11.8 \%(n=17)$; the negative samples $(n=127)$ were tested for rubella with $3.9 \%(n=5)$ positive results. Among those non reactive for rubella $(n=122)$ tests were done for leptospirosis and hantavirus. Positive tests for leptospirosis were $13.9 \%(\mathrm{n}=17)$ and none for hantavirus. Non reactive results $(70.8 \%)$ were considered as indefinite febrile illness. Low schooling was associated with dengue, rubella and leptospirosis, dyspnea with both dengue and leptospirosis and exanthema/ petechia with dengue and rubella.

OLIVEIRA \& PONTES studied the frequencies of immunity against hepatitis $\mathrm{B}(\mathrm{HB})$ and of potentially contaminating accidents among medical students of a Brazilian public university. Of all the 400 students who should have been immunized $303(75.7 \%)$ answered an anonymous, selfadministered questionnaire. The frequency of immunity among women was higher than that among men. The results from this study showed that: 1 . the frequency of immunity against HB is high among the evaluated medical students, although verification of response to vaccination is not a concern for them; 2. anti-HBs titers should be verified after complete vaccination; 3. the frequency of potentially contaminating accidents is high.

INOUE et al. reported a case of hematopoietic stem cell transplant in which the donor had a history of previous malaria. He presented Plasmodium vivax in thick blood smears one month after transplant and was treated accordingly. The polymerase chain reaction (PCR) was able to detect malaria infection in the donor one week earlier than thick blood film. Authors highlight the importance of monitoring recipients and donors in transplant procedures with the aim of reducing the risk of malaria transmission.

LIMA et al. studied the parasite density in canine leishmaniasis (Leishmania infantum chagasi) in tissues of symptomatic and asymptomatic seropositive dogs. There was no difference in parasite density in the skin and lymph nodes between the two groups and these results strongly suggest that both symptomatic and asymptomatic seropositive dogs can serve as a source of infection, principally considering the high number of parasites detected in the skin, the place where the vector L. longipalpis takes its blood meal.

MENTZ et al. reported on the susceptibility and morbidity between male and female Swiss mice infected with Angiostrongylus costaricensis and the results indicate that there are no significant differences related to gender of the host, except for higher length of worms developed in male mice.

PELLEGRINO et al. reported a ring enhancing intracranial lesion in an HIV-infected patient which posed a differential diagnosis between cerebral toxoplasmosis and a tuberculoma. Only a trial with antituberculous drugs proved to be a valuable strategy to infer the correct diagnosis of tuberculoma in this patient.

Thales de BRITO, Editor Pedro Paulo CHIEFFI, Associate Editor Marcello F. FRANCO, Associate Editor 